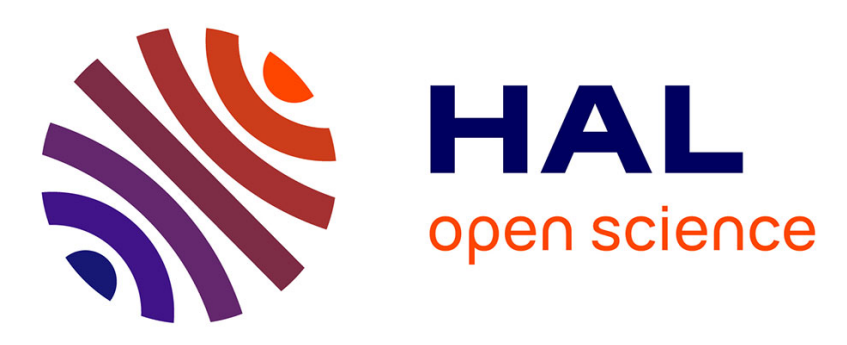

\title{
Towards Anomaly-Tolerant Systems by Dissipation Block Synthesis
}

\author{
Arthur Perodou, Christophe Combastel, Ali Zolghadri
}

\section{To cite this version:}

Arthur Perodou, Christophe Combastel, Ali Zolghadri. Towards Anomaly-Tolerant Systems by Dissipation Block Synthesis. 5th International Conference on Control and Fault-Tolerant Systems (IEEE SysTol), Sep 2021, Saint-Raphaël, France. hal-03370194

\section{HAL Id: hal-03370194 \\ https://hal.science/hal-03370194}

Submitted on 7 Oct 2021

HAL is a multi-disciplinary open access archive for the deposit and dissemination of scientific research documents, whether they are published or not. The documents may come from teaching and research institutions in France or abroad, or from public or private research centers.
L'archive ouverte pluridisciplinaire $\mathbf{H A L}$, est destinée au dépôt et à la diffusion de documents scientifiques de niveau recherche, publiés ou non, émanant des établissements d'enseignement et de recherche français ou étrangers, des laboratoires publics ou privés. 


\title{
Towards Anomaly-Tolerant Systems by Dissipation Block Synthesis
}

\author{
Arthur Perodou, Christophe Combastel and Ali Zolghadri, Senior Member, IEEE
}

\begin{abstract}
The complexity of modern control systems requires mitigating the effects of numerous anomalies, such as attacks, faults and network-induced disturbances. In this perspective, the synthesis problem of a dissipation block for anomaly-tolerant control is addressed. The dissipation technique consists in interconnecting a block to a system in order to make their interconnection dissipative. The underlying idea is that, if the resulting dissipative characterization is suitably chosen, the considered anomalies can be mitigated. In this paper, it is revealed how the block, here an LTI system, can be synthesized by deriving sufficient LMI-based conditions. A numerical example is provided to illustrate this result which is applied to design a control reconfiguration, providing a defense mechanism that ensures resilience with respect to a data-injection cyber attack.
\end{abstract}

Keywords : anomaly tolerant control, dissipation block, passivation, QSR-dissipative systems

\section{INTRODUCTION}

The ever-increasing integration of cyber elements to physical systems offers new computational and interconnection capabilities, but also leads the design of modern control systems to be a challenging problem. Part of this complexity comes from the many sources of anomaly that have to be considered and mitigated, including attacks, faults and network-induced disturbances [1], [2], [3].

In order to design a control system tolerant to anomalies, two complementary strategies are commonly employed, respectively referred to passive and active in the faultmanagement literature [4]. The first strategy consists in designing a system such that it inherently satisfies some specifications even in abnormal conditions, and is particularly linked with usual Robust Control techniques [5]. In the second strategy, the system is monitored and reconfigured when an anomaly occurs, and is based on a detection and reconfiguration scheme [2].

Passivity is an ubiquitous concept in System and Control theory [6]. First, passive systems inherit important properties such as stability and minimum-phase. Moreover, passive systems can be represented by structured models that make their analysis, control and design easier [6], [7]. Last but not least, the compositional feature of passive systems appears promising for modern control systems [8]. Dissipative theory

The authors are with Univ. Bordeaux, IMS-lab, CNRS UMR 5218, Bat. A31, 351 cours de la Libération, 33400, Talence, France. e-mail: \{arthur.perodou, christophe.combastel, ali.zolghadri\}@ims-bordeaux. fr

This study has been carried out with financial support from the French National Research Agency (ANR) in the framework of the Investments for the Future Programme IdEx Bordeaux-SysNum (ANR-10-IDEX-03-02). The financial support from the "Conseil Régional de la Nouvelle-Aquitaine" is also gratefully acknowledged. may be viewed as an extension of these properties for a larger class of interconnected systems [9], [10].

From an anomaly-tolerant control perspective, a promising approach that can be used in both passive and active strategies is based on the concept of dissipation block. This consists of interconnecting a block to a system in order to make their interconnection dissipative. If the dissipation block is suitably chosen, this enables to mitigate the effects of anomalies on the control system. For instance, the introduction of a particular block based on the wave variable transformation [8] enables to ensure the $\mathcal{L}_{2}$-stability of passive systems interconnected over a network subject to delays and packet loss [11] or denial-of-service attacks [12]. Another relevant example is the block insertion based on the $M$-transformation matrix [13] that passivates, with a desired passivity index, the interconnection of $M$ with a stable system $G$ in order to obtain a system tolerant to anomalies such as quantization [8], delays [14], denial-of-service [15] or data injection [16] attacks. In those works, the blocks are structured static gains and their synthesis is generally complex. Recently, it has been proposed in [17] a method based on Linear Matrix Inequality (LMI) optimization [18] to synthesize an unstructured linear time-invariant (LTI) system $M$ for fault-tolerant purposes. Based on the passivity indices of a faulty system $G$, the block $M$ is synthesized to ensure the asymptotic stability of their interconnection.

In this paper, the problem of synthesizing an LTI system $M$ such that its interconnection $G \star M$ with a given system $G$ satisfies a given dissipative characterization is addressed. In particular, it is revealed that this problem can be solved using LMI-based sufficient conditions. In addition to this numerical advantage, the main contribution of this paper comes from the generality of the result. Indeed, a wide range of dissipative characterizations for the system $G$ and the interconnection $G \star M$ are allowed. Moreover, this methods allows to synthesize a general LTI system $M$, that admits structured or static blocks as particular cases.

The paper is organized as follows. First, brief reminders on the dissipative characterization are provided in Section II. In Section III, the dissipation block synthesis problem is explicitly formulated. In Section IV, it is shown how this problem can be solved using LMI-based conditions. Finally, an illustrative example is provided in Section VI, while Section VI provides some concluding remarks.

\section{PRELIMINARIES}

\section{A. Notations}

Lower (upper) case letters are used for vectors (matrices). $\mathbb{R}^{n \times m}$ denotes the set of real-valued matrices of size $n \times m$, 
$\mathbb{S}_{n}$ the set of real symmetric matrices of size $n \times n$ and $\mathbb{D}_{n}$ the set of diagonal real-valued matrices of size $n \times n$. $I_{n}$ and $0_{n \times m}$ are respectively the identity matrix of $\mathbb{R}^{n \times n}$ and the zero matrix of $\mathbb{R}^{n \times m}$. The subscripts are omitted when obvious from the context. $X^{T}$ stands for transpose of $X$ while $M>$ (resp. $\geq) 0$ denotes positive (semi-) definiteness, and $\operatorname{diag}(v)$ is the diagonal matrix with the elements of vector $v$ on the diagonal. Bold characters denote either explicit decision variables in a design problem or optimization variables in an optimization problem. Signals are assumed to belong to $\mathcal{L}_{2 e}$, the extended space of $\mathcal{L}_{2}[0, \infty)$.

\section{B. Background}

In this paper, the input-output characterization of dissipative systems of [9] is used.

Definition 1:

Let $Q \in \mathbb{S}_{p}, S \in \mathbb{R}^{p \times m}$ and $R \in \mathbb{S}_{m}$ be three given matrices. The system $G: u \rightarrow y$ is $\{Q, S, R\}$-dissipative if

$$
\forall u \in \mathcal{L}_{2 e}, \forall T \geq 0, \int_{0}^{T}\left[\begin{array}{l}
y(t) \\
u(t)
\end{array}\right]^{T}\left[\begin{array}{cc}
Q & S \\
S^{T} & R
\end{array}\right]\left[\begin{array}{c}
y(t) \\
u(t)
\end{array}\right] d t \geq 0
$$

This definition includes several usual characterization. For instances, a system is passive if it is $\left\{0, \frac{1}{2} I, 0\right\}$ dissipative, has finite gain if it is $\left(-I, 0, \gamma^{2} I\right)$-dissipative, is input-feedforward-output-feedback passive (IF-OFP) if it is $\left(-\rho I, \frac{1}{2} I,-\nu I\right)$ with $\rho, \nu \in \mathbb{R}$, and is very strictly passive (VSP) if additionally $\rho>0, \nu>0$ [13]. It is worth to notice that certain properties can be deduced from a $\{Q, S, R\}$-dissipative characterization of a system. For instance, if $Q<0$ then the system $G$ is finite-gain stable, and if additionally $G$ has a zero-state detectable state-space realization, then $G$ is asymptotically stable [9].

Remark 1: The problem of finding a relevant $\{Q, S, R\}-$ dissipative characterization of a system $G$ is highly contextual and is still under investigation. For instances, the reader may refer to [9, Chap. 8-9] for frequency domain criteria for linear systems and general conditions for simple nonlinear systems to test dissipativeness, to [8, Sec. 6] for an introduction to experimental determination of passivity indices, or to [17, Remark 3] for a brief overview of recent results on the estimation of dissipative properties.

\section{Problem Statement}

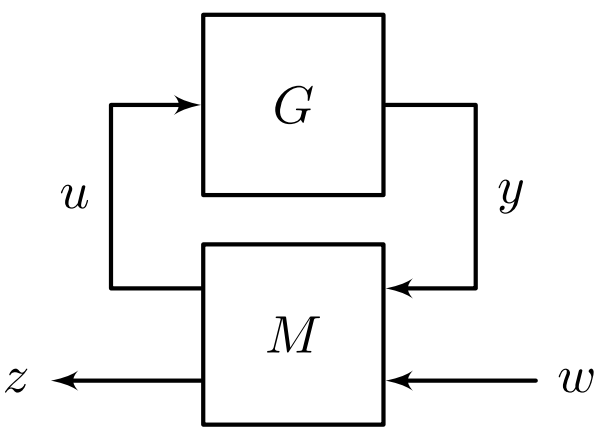

Fig. 1. Interconnection of $G$ with the dissipation block $M$
Consider a system $G$ with input $u$ and output $y$

$$
y=G(u)
$$

In the sequel, $G$ is only characterized by its dissipative characterization. This description is general and includes, for instance, non-linear and uncertain systems.

The system $G$ is considered to be subject to anomalies that depend on the context and that may be faults, attacks or network-induced disturbances. In order to mitigate the effects of the considered anomalies, it is interconnected with an LTI system $M$ whose model is described as follows

$$
(M):\left\{\begin{array}{l}
\dot{x}(t)=A x(t)+B_{y} y(t)+B_{w} w(t) \\
u(t)=C_{u} x(t)+D_{u y} y(t)+D_{u w} w(t) \\
z(t)=C_{z} x(t)+D_{z y} y(t)+D_{z w} w(t)
\end{array}\right.
$$

where $A \in \mathbb{R}^{n \times n}, B_{y} \in \mathbb{R}^{n \times p}, B_{w} \in \mathbb{R}^{n \times m}, C_{u} \in \mathbb{R}^{m \times n}$, $D_{u y} \in \mathbb{R}^{m \times p}, D_{u w} \in \mathbb{R}^{m \times m}, C_{z} \in \mathbb{R}^{p \times n}, D_{z y} \in \mathbb{R}^{p \times p}$, $D_{z w} \in \mathbb{R}^{p \times m}$. In addition, it is assumed that $x(0)=0$.

The interconnected systems $G \star M$ is then described as

$$
(G \star M):\left\{\begin{aligned}
y(t) & =G(u)(t) \\
{\left[\begin{array}{c}
\dot{x}(t) \\
u(t)
\end{array}\right] } & =\left[\begin{array}{cc}
A & B_{y} \\
C_{u} & D_{u y}
\end{array}\right]\left[\begin{array}{l}
x(t) \\
y(t)
\end{array}\right]+\left[\begin{array}{c}
B_{w} \\
D_{u w}
\end{array}\right] w(t) \\
z(t) & =\left[\begin{array}{ll}
C_{z} & D_{z y}
\end{array}\right]\left[\begin{array}{l}
x(t) \\
y(t)
\end{array}\right]+D_{z w} w(t)
\end{aligned}\right.
$$

Problem 1:

Given a $\left\{Q_{G}, S_{G}, R_{G}\right\}$-dissipative system $G$, find if there exists an LTI system $M$ described by (1) such that the resulting interconnection $G \star M$ is $\left\{Q_{p}, S_{p}, R_{p}\right\}$-dissipative. If so, compute it.

\section{MAIN RESUlT}

In order to tackle Problem 1, the sub-problem of finding a system $M$ with fixed order $n$ is first tackled. Then, it is proposed to iteratively increment $n$, with the aim of finding its minimum value.

\section{A. Synthesis with fixed order}

In this subsection, it is assumed that the order $n$ of $M$ is fixed. First, next lemma provides an LMI feasibility problem as a sufficient condition for the analysis problem of checking if $G \star M$ is $\left\{Q_{p}, S_{p}, R_{p}\right\}$-dissipative.

Lemma 1:

Let $Q_{p} \in \mathbb{S}_{p}, S_{p} \in \mathbb{R}^{p \times m}, Q_{p} \in \mathbb{S}^{m}$ be given matrices. Let $G$ be a $\left\{Q_{G}, S_{G}, R_{G}\right\}$-dissipative system. Let $M$ be given as in (1) where $n$ is known.

Then, the interconnected system $G \star M$ described by (2) is $\left\{Q_{p}, S_{p}, R_{p}\right\}$-dissipative if $\exists \boldsymbol{P} \in \mathbb{S}_{n}, \exists \boldsymbol{\tau} \in \mathbb{R}$,

$$
\boldsymbol{P}>0 \quad \boldsymbol{\tau}>0
$$




$$
F^{T}\left[\begin{array}{cccccc}
0 & 0 & -\boldsymbol{P} & 0 & 0 & 0 \\
0 & -\boldsymbol{\tau} R_{G} & 0 & -\boldsymbol{\tau} S_{G}^{T} & 0 & 0 \\
-\boldsymbol{P} & 0 & 0 & 0 & 0 & 0 \\
0 & -\boldsymbol{\tau} S_{G} & 0 & -\boldsymbol{\tau} Q_{G} & 0 & 0 \\
0 & 0 & 0 & 0 & Q_{p} & S_{p} \\
0 & 0 & 0 & 0 & S_{p}^{T} & R_{p}
\end{array}\right] F \geq 0
$$

where $F:=\left[\begin{array}{cc}F_{1}^{T} & F_{2}^{T}\end{array}\right]^{T}$ and

$F_{1}:=\left[\begin{array}{ccc}A & B_{y} & B_{w} \\ C_{u} & D_{u y} & D_{u w} \\ I & 0 & 0 \\ 0 & I & 0\end{array}\right], F_{2}:=\left[\begin{array}{ccc}C_{z} & D_{z y} & D_{z w} \\ 0 & 0 & I\end{array}\right]$

Proof:

Consider the signals $u, x, w \in \mathcal{L}_{2 e}$ as in (2). Observe that $\forall t \in \mathbb{R}$,

$F\left[\begin{array}{c}x(t) \\ y(t) \\ w(t)\end{array}\right]=\left[\begin{array}{llllll}\dot{x}^{T}(t) & u^{T}(t) & x^{T}(t) & y^{T}(t) & z^{T}(t) & w^{T}(t)\end{array}\right]^{T}$

Pre- and post-multiplying (3) by $\left[\begin{array}{llll}x^{T}(t) & y^{T}(t) & w^{T}(t)\end{array}\right]$ and its transpose conjugate leads then to:

$$
\begin{aligned}
{\left[\begin{array}{c}
z(t) \\
w(t)
\end{array}\right]^{T}\left[\begin{array}{ll}
Q_{p} & S_{p} \\
S_{p}^{T} & R_{p}
\end{array}\right] } & {\left[\begin{array}{c}
z(t) \\
w(t)
\end{array}\right]-2 \dot{x}^{T}(t) \boldsymbol{P} x(t) } \\
& -\boldsymbol{\tau}\left[\begin{array}{c}
y(t) \\
u(t)
\end{array}\right]^{T}\left[\begin{array}{ll}
Q_{G} & S_{G} \\
S_{G}^{T} & R_{G}
\end{array}\right]\left[\begin{array}{c}
y(t) \\
u(t)
\end{array}\right] \geq 0
\end{aligned}
$$

Let $T \geq 0$. By integration of last inequality between $t=0$ and $T$, it comes that $G \star M$ is $\left\{Q_{p}, S_{p}, R_{p}\right\}$-dissipative as:

$$
\begin{array}{r}
-2 \int_{0}^{T} \dot{x}^{T}(t) \boldsymbol{P} x(t) d t \leq 0 \\
-\boldsymbol{\tau} \int_{0}^{T}\left[\begin{array}{l}
y(t) \\
u(t)
\end{array}\right]^{T}\left[\begin{array}{ll}
Q_{G} & S_{G} \\
S_{G}^{T} & R_{G}
\end{array}\right]\left[\begin{array}{l}
y(t) \\
u(t)
\end{array}\right] d t \leq 0
\end{array}
$$

Remark 2: The introduction of the optimization variables $\boldsymbol{P}$ and $\boldsymbol{\tau}$, that are called multipliers, is closely related with the so-called S-procedure. The interested reader may refer to [18, Chap. 8] for instance.

Based on Lemma 1, and the application of the Schur lemma [18, Chap. 2], the next theorem reveals that a similar LMI-based sufficient condition enables to solve Problem 1.

\section{Theorem 1:}

Let $Q_{p} \in \mathbb{S}_{p}, S_{p} \in \mathbb{R}^{p \times m}, R_{p} \in \mathbb{S}^{m}$ be given matrices, where $Q_{p} \leq 0$. Let $G$ be a $\left\{Q_{G}, S_{G}, R_{G}\right\}$-dissipative system with $R_{G} \geq 0$. Let $M$ be given as in (1) where $n$ is known. Denote $r_{p}:=\operatorname{rank}\left(Q_{p}\right)$ and $r_{G}:=\operatorname{rank}\left(R_{G}\right)$ and factorize $Q_{p}$ and $R_{G}$ as

$$
Q_{p}=V_{p}^{T} \Lambda_{p} V_{p} \quad R_{G}=V_{G}^{T} \Lambda_{G} V_{G}
$$

with $\Lambda_{p} \in \mathbb{D}_{r_{p}}, V_{p} \in \mathbb{R}^{r_{p} \times m}, \Lambda_{G} \in \mathbb{D}_{r_{G}}$ and $V_{G} \in \mathbb{R}^{r_{G} \times p}$. Then $(i) \Rightarrow(i i)$. (i) $\exists \boldsymbol{A} \in \mathbb{R}^{n \times n}, \boldsymbol{B}_{\boldsymbol{y}} \in \mathbb{R}^{n \times p}, \boldsymbol{B}_{\boldsymbol{w}} \in \mathbb{R}^{n \times m}, \boldsymbol{C}_{\boldsymbol{u}_{\boldsymbol{\tau}}} \in$ $\mathbb{R}^{m \times n}, \boldsymbol{D}_{\boldsymbol{u} \boldsymbol{y}_{\boldsymbol{\tau}}} \in \mathbb{R}^{m \times p}, \boldsymbol{D}_{\boldsymbol{u} \boldsymbol{w}_{\boldsymbol{\tau}}} \in \mathbb{R}^{m \times m}, \boldsymbol{C}_{\boldsymbol{z}} \in \mathbb{R}^{p \times n}$, $\boldsymbol{D}_{\boldsymbol{z} \boldsymbol{y}} \in \mathbb{R}^{p \times p}, \boldsymbol{D}_{\boldsymbol{z w}} \in \mathbb{R}^{p \times m}, \boldsymbol{\tau} \in \mathbb{R}$ such that

$$
\left[\begin{array}{ccc}
\Theta & \boldsymbol{\tau}>0 \\
\Phi_{G} & \Phi_{p} \\
\Phi_{p}^{T} & \Gamma_{G} & 0 \\
\Gamma & 0 & \Gamma_{p}
\end{array}\right] \geq 0
$$

$$
\begin{aligned}
\text { hold where } \Theta & :=\left[\begin{array}{lll}
\theta_{11} & \theta_{12} & \theta_{13} \\
\theta_{12}^{T} & \theta_{22} & \theta_{23} \\
\theta_{13}^{T} & \theta_{23}^{T} & \theta_{33}
\end{array}\right], \\
\theta_{11} & :=-\boldsymbol{A}-\boldsymbol{A}^{\boldsymbol{T}} \\
\theta_{12} & :=-\boldsymbol{B}_{\boldsymbol{y}}-\boldsymbol{C}_{\boldsymbol{u}_{\boldsymbol{\tau}}}^{\boldsymbol{T}} S_{G}^{T} \\
\theta_{13} & :=-\boldsymbol{B}_{\boldsymbol{w}}+\boldsymbol{C}_{\boldsymbol{z}}^{\boldsymbol{T}} S_{p} \\
\theta_{22} & :=-\boldsymbol{D}_{\boldsymbol{u} \boldsymbol{y}_{\boldsymbol{\tau}}}^{\boldsymbol{T}} S_{G}^{T}-S_{G} \boldsymbol{D}_{\boldsymbol{u} \boldsymbol{y}_{\boldsymbol{\tau}}}-\boldsymbol{\tau} Q_{G} \\
\theta_{23} & :=-S_{G} \boldsymbol{D}_{\boldsymbol{u}}+\boldsymbol{D}_{\boldsymbol{z} \boldsymbol{y}}^{\boldsymbol{T}} S_{p} \\
\theta_{33} & :=\boldsymbol{D}_{\boldsymbol{z} \boldsymbol{w}}^{\boldsymbol{T}} S_{p}+S_{p}^{T} \boldsymbol{D}_{\boldsymbol{z} \boldsymbol{w}}+R_{p}
\end{aligned}
$$

and

1) If $r_{G} \geq 1$ and $r_{p} \geq 1$, then

$$
\begin{gathered}
\Phi_{G}^{T}=V_{G}\left[\begin{array}{lll}
\boldsymbol{C}_{\boldsymbol{u}_{\boldsymbol{\tau}}} & \boldsymbol{D}_{\boldsymbol{u} \boldsymbol{y}_{\boldsymbol{\tau}}} & \boldsymbol{D}_{\boldsymbol{u} \boldsymbol{w}_{\boldsymbol{\tau}}}
\end{array}\right] \quad \Gamma_{G}=\boldsymbol{\tau} \Lambda_{G}^{-1} \\
\Phi_{p}^{T}=V_{p}\left[\begin{array}{lll}
\boldsymbol{C}_{\boldsymbol{z}} & \boldsymbol{D}_{\boldsymbol{z y}} & \boldsymbol{D}_{\boldsymbol{z w}}
\end{array}\right] \quad \Gamma_{p}=-\Lambda_{p}^{-1}
\end{gathered}
$$

2) If $r_{G} \geq 1$ and $r_{p}=0$, then (6), $\Phi_{p}=0$ and $\Gamma_{p}=0$.

3) If $r_{G}=0$ and $r_{p} \geq 1$, then $\Phi_{G}=0, \Gamma_{G}=0$ and (7).

4) If $r_{G}=r_{p}=0$, then $\Phi_{G}=0, \Gamma_{G}=0, \Phi_{p}=0$ and $\Gamma_{p}=0$.

(ii) There exists a solution to Problem 1, that is there exists a system $M$ defined by (1) such that $G \star M$ is $\left\{Q_{p}, S_{p}, R_{p}\right\}$-dissipative, where $A, B_{y}, B_{w}, C_{z}, D_{z y}$ and $D_{z w}$ are directly obtained from condition $(i)$ and

$$
\left[C_{u}\left|D_{u y}\right| D_{u w}\right]:=\tau^{-1}\left[C_{u_{\tau}}\left|D_{u y_{\tau}}\right| D_{u w_{\tau}}\right]
$$

Proof:

For the sake of brevity, as the proofs of cases 2), 3) and 4) can be straightly derived from case 1), it is assumed that $r_{G} \geq 1$ and $r_{p} \geq 1$.

By Lemma 1, a sufficient condition for $(i i)$ is provided by $\exists A, B_{y}, B_{w}, C_{u}, D_{u y}, D_{u w}, C_{z}, D_{z y}, D_{z w}, \exists P>0$, $\exists \tau>0$, such that (3) holds.

Denote $\boldsymbol{T}$ such that $P=T^{T} \boldsymbol{T}$. Define $A_{T}:=\boldsymbol{T} \boldsymbol{A} \boldsymbol{T}^{-1}$, $B_{y_{T}}:=T B_{y}, B_{w_{T}}:=T B_{w}, C_{u_{T}}:=C_{u} T^{-1}$ and $C_{z_{T}}:=C_{z} T^{-1}$. This corresponds to the change of state variable $x_{T}:=T x$ for the system $M$. It is then equivalent to synthesize these new representation matrices.

By pre- and post-multiplying (3) by

$$
\left[\begin{array}{ccc}
\boldsymbol{T}^{-1} & 0 & 0 \\
0 & I & 0 \\
0 & 0 & I
\end{array}\right]^{T} \text { and }\left[\begin{array}{ccc}
\boldsymbol{T}^{-1} & 0 & 0 \\
0 & I & 0 \\
0 & 0 & I
\end{array}\right]
$$


the inequality (3) is equivalent by congruence [19] to

$$
\begin{aligned}
& {\left[\begin{array}{ccc}
0 & 0 & \boldsymbol{C}_{\boldsymbol{z}_{T}}^{\boldsymbol{T}} S_{p} \\
0 & 0 & \boldsymbol{D}_{\boldsymbol{z} \boldsymbol{y}}^{\boldsymbol{T}} S_{p} \\
S_{p}^{T} \boldsymbol{C}_{\boldsymbol{z}_{\boldsymbol{T}}} & S_{p}^{T} \boldsymbol{D}_{\boldsymbol{z} \boldsymbol{y}} & \boldsymbol{D}_{\boldsymbol{z} \boldsymbol{w}}^{\boldsymbol{T}} S_{p}+S_{p}^{T} \boldsymbol{D}_{\boldsymbol{z w}}+R_{p}
\end{array}\right]} \\
& -\left[\begin{array}{ccc}
A_{T}+A_{T}^{T} & B_{y_{T}} & B_{w_{T}} \\
B_{y_{T}}^{T} & 0 & 0 \\
B_{w_{T}}^{T} & 0 & 0
\end{array}\right] \\
& \boldsymbol{- \tau}\left[\begin{array}{ccc}
0 & \boldsymbol{C}_{\boldsymbol{u}_{T}}^{\boldsymbol{T}} S_{G}^{T} & 0 \\
S_{G} \boldsymbol{C}_{\boldsymbol{u}_{\boldsymbol{T}}} & \boldsymbol{D}_{\boldsymbol{u} \boldsymbol{y}}^{\boldsymbol{T}} S_{G}^{T}+S_{G} \boldsymbol{D}_{\boldsymbol{u} \boldsymbol{y}}+Q_{G} & S_{G} \boldsymbol{D}_{\boldsymbol{u w}} \\
0 & \boldsymbol{D}_{\boldsymbol{u} \boldsymbol{w}}^{\boldsymbol{T}} S_{G}^{T} & 0
\end{array}\right]
\end{aligned}
$$

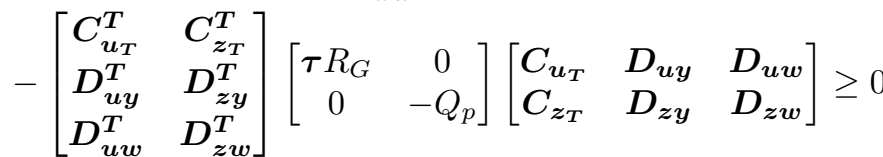

Define $\boldsymbol{C}_{\boldsymbol{u}_{\tau}}:=\tau \boldsymbol{C}_{\boldsymbol{u}_{T}}, \boldsymbol{D}_{\boldsymbol{u} \boldsymbol{y}_{\tau}}:=\tau \boldsymbol{D}_{\boldsymbol{u y}}, \boldsymbol{D}_{\boldsymbol{u} \boldsymbol{w}_{\tau}}:=\tau \boldsymbol{D}_{\boldsymbol{u w}}$. Using (4) and by applying the Schur Lemma [18, Chap. 2], last inequality is equivalent to

$$
\left[\begin{array}{ccc}
\Theta & \boldsymbol{\tau}^{-1} \Phi_{G} & \Phi_{p} \\
\boldsymbol{\tau}^{-1} \Phi_{G}^{T} & \boldsymbol{\tau}^{-1} \Lambda_{G}^{-1} & 0 \\
\Phi_{p}^{T} & 0 & -\Lambda_{p}^{-1}
\end{array}\right] \geq 0
$$

Finally, by congruence, last inequality is equivalent to (5) by pre- and post- multiplying by

$$
\left[\begin{array}{ccc}
I & 0 & 0 \\
0 & \tau I & 0 \\
0 & 0 & I
\end{array}\right]
$$

Therefore, by successive equivalence and the application of Lemma 1, condition $(i i)$ is satisfied.

Remark 3: The factorizations (4) can be obtained using an eigenvalue decomposition. For instance, as $Q_{G} \leq 0$ is a real symmetric matrix, $\exists U_{G} \in \mathbb{R}^{p \times p}, \exists \Lambda_{G}<0 \in \mathbb{D}_{r_{G}}$,

$$
Q_{G}=U_{G}^{T}\left[\begin{array}{cc}
\Lambda_{G} & 0 \\
0 & 0
\end{array}\right] U_{G}=U_{G}^{T}\left[\begin{array}{ll}
I & 0
\end{array}\right]^{T} \Lambda_{G}\left[\begin{array}{ll}
I & 0
\end{array}\right] U_{G}
$$

The matrix $V_{G}$ is then defined by $V_{G}:=\left[\begin{array}{ll}I & 0\end{array}\right] U_{G}$.

Theorem 1 provides a sufficient LMI-based condition for the synthesis of an unstructured LTI system $M$. However, it should be noticed that, if needed, condition $(i)$ can be specialized to a system $M$ with some particular structure. For instance, the wave variable transformation [8] may be obtained with the following constraints

$$
\left[\begin{array}{ccc}
A & B_{y} & B_{w} \\
C_{u} & D_{u y} & D_{u w} \\
C_{z} & D_{z y} & D_{z w}
\end{array}\right]=\left[\begin{array}{ccc}
0 & 0 & 0 \\
0 & b I & -\sqrt{2 b} I \\
0 & \sqrt{2 b} I & -I
\end{array}\right]
$$

while the M-transformation matrix [13] is given by

$$
\left[\begin{array}{ccc}
A & B_{y} & B_{w} \\
C_{u} & D_{u y} & D_{u w} \\
C_{z} & D_{z y} & D_{z w}
\end{array}\right]=\left[\begin{array}{ccc}
0 & 0 & 0 \\
0 & -m_{f} I & I \\
0 & \left(m_{s}-m_{p} m_{f}\right) I & m_{p} I
\end{array}\right]
$$

\section{B. On minimum-order dissipation block $M$}

In Theorem 1, the order $n$ of $M$ is considered fixed. An associate problem is to find $M$ with minimum order. This may be achieved using an iterative algorithm that increments the order of $n$ and solves the associate LMI feasibility problem at each iteration until a feasible solution is found.

\section{ILlustration}

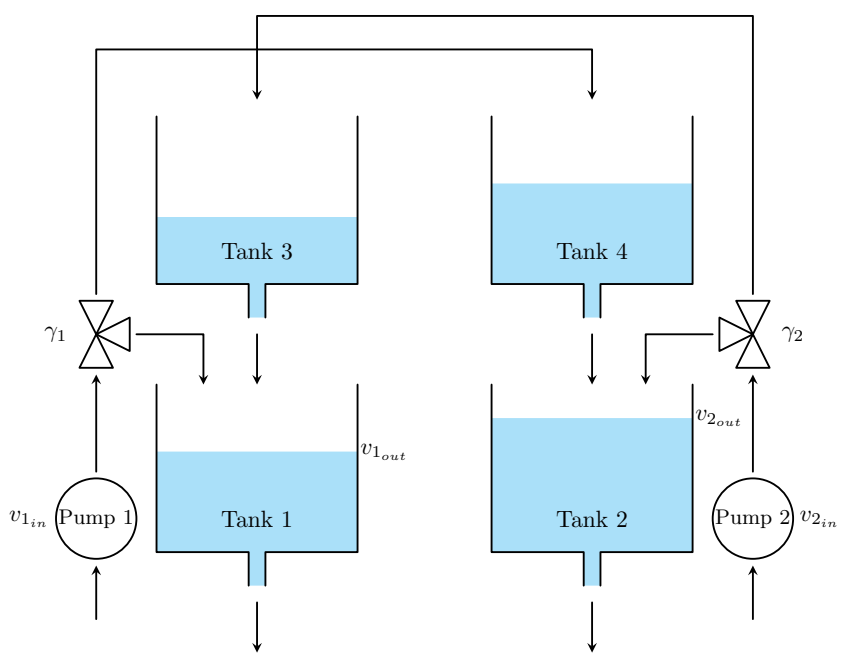

Fig. 2. Quadruple water-tank process

In this section, a numerical example is provided to illustrate the main result of this paper and the potential of the dissipation block approach.

The quadruple-tank process testbed introduced in [20] is considered (Fig. 2). The process inputs are the voltages $v_{1_{\text {in }}}$ and $v_{2_{i n}}$ applied to the pumps, and the outputs are the voltages $v_{1_{\text {out }}}$ and $v_{2_{\text {out }}}$ from level measurement devices of tanks 1 and 2. The process is modeled as follows:

$$
\left\{\begin{aligned}
\dot{h}(t) & =A_{p} \sqrt{h(t)}+B_{p} v_{i n}(t) \\
v_{\text {out }}(t) & =C_{p} h(t)
\end{aligned}\right.
$$

where $h=\left[\begin{array}{llll}h_{1} & h_{2} & h_{3} & h_{4}\end{array}\right]^{T}, h_{i}$ the water level of tank $i$, $v_{\text {in }}=\left[\begin{array}{ll}v_{1_{\text {in }}} & v_{2_{\text {in }}}\end{array}\right]^{T}, v_{\text {out }}=\left[\begin{array}{ll}v_{1_{\text {out }}} & v_{2_{\text {out }}}\end{array}\right]^{T}$ and

$$
\begin{aligned}
A_{p} & =\left[\begin{array}{cccc}
-0.1123 & 0 & 0.1123 & 0 \\
0 & -0.0789 & 0 & 0.0789 \\
0 & 0 & -0.1123 & 0 \\
0 & 0 & 0 & -0.0789
\end{array}\right] \\
B_{p}= & {\left[\begin{array}{cc}
0.0833 & 0 \\
0 & 0.0624 \\
0 & 0.0476 \\
0.0312 & 0
\end{array}\right] C_{p}=\left[\begin{array}{cccc}
0.5 & 0 & 0 & 0 \\
0 & 0.5 & 0 & 0
\end{array}\right] }
\end{aligned}
$$

Due to physical limitations, the inputs $v_{i_{i n}}, i=1,2$, are such that $v_{\min } \leq v_{i_{i n}} \leq v_{\max }$ where $v_{\min }=0 \mathrm{~V}$ and $v_{\max }=12 \mathrm{~V}$. Moreover, for safety reasons, the water tanks should not overflow nor be empty. The safety set $\mathcal{S}$ is then

$$
\mathcal{S}:=\left\{h \mid \forall i=1, \ldots, 4, \quad h_{\min } \leq h_{i} \leq h_{\max }\right\}
$$


where $h_{\min }=0 \mathrm{~cm}$ and $h_{\max }=15 \mathrm{~cm}$. The process is controlled by remote PI controllers, using a wireless network, that compute the control input $v_{c}$ from a reference $r$ and measurement $v_{\text {out }}$ such as $v_{i_{c}}=K_{i}\left(1+\frac{1}{T_{i} \cdot s}\right)\left(r_{i}-v_{i_{\text {out }}}\right)$ where $K_{1}=3, K_{2}=2.7, T_{1}=30 \mathrm{~s}$ and $T_{2}=40 \mathrm{~s}$.

\section{A. Uncertain linear approximation}

In this section, it is proposed to approximate the process by an uncertain linear system $G$. To achieve this, introduce the variables $x_{G}:=h-h^{0}$ and $u:=v_{i n}-v_{i n}^{0}$ where $\left(h^{0}, v_{i n}^{0}\right)$ is a stationary operating point of the process, that is

$$
0=A_{p} \sqrt{h^{0}}+B_{p} v_{i n}^{0}
$$

Using a Taylor-Mclaurin series expansion, it comes that for $h_{i}$ in the neighborhood of $h_{i}^{0}$

$\sqrt{x_{G_{i}}+h_{i}^{0}}=\sqrt{h_{i}^{0}} \sqrt{1+\frac{x_{G_{i}}}{h_{i}^{0}}}=\sqrt{h_{i}^{0}}\left(1+\frac{x_{G_{i}}}{2 h_{i}^{0}}+\delta_{i} x_{G_{i}}\right)$

where $\delta_{i} \in\left[c_{\delta_{i}}-r_{\delta_{i}}, c_{\delta_{i}}+r_{\delta_{i}}\right]$ is a bounded uncertain parameter. Denote $A_{G}:=\frac{1}{2} A_{p}\left(h_{\text {diag }}^{0}\right)^{-1 / 2}, A_{\Delta}:=A_{p} \sqrt{h_{\text {diag }}^{0}}$, $h_{\text {diag }}^{0}=\operatorname{diag}\left(h^{0}\right)$ and $\Delta:=\operatorname{diag}(\delta)$. By introducing $y(t):=$ $v_{\text {out }}(t)-C_{p} h^{0}$, a linear approximation is then given by

$$
(G):\left\{\begin{aligned}
\dot{x}_{G}(t) & =\left(A_{G}+A_{\Delta} \Delta\right) x_{G}(t)+B_{p} u(t) \\
y(t) & =C_{p} x_{G}(t)
\end{aligned}\right.
$$

In addition, define $C_{\Delta}:=\operatorname{diag}\left(c_{\delta}\right)$ and $R_{\Delta}:=\operatorname{diag}\left(r_{\delta}\right)$.

\section{B. Dissipative characterization}

In this subsection, a method is proposed to compute a $\left\{q_{G} I, s_{G} I, r_{G} I\right\}$-dissipative characterization of $G$, where $q_{G}, s_{G}, r_{G} \in \mathbb{R}$ and $q_{G}<0$. When $G$ is a single-inputsingle-output LTI system, this characterizes a disk of center $c_{d}$ and radius $r_{d}$ in the complex plane [9], where

$$
c_{d}:=\left(-\frac{s_{G}}{q_{G}}, 0\right) \quad r_{d}:=\sqrt{\frac{s_{G}^{2}-q_{G} r_{G}}{q_{G}^{2}}}
$$

In this case, minimizing $r_{d}^{2}$ while $G$ being $\left\{q_{G}, s_{G}, r_{G}\right\}$ dissipative leads to the smallest disk which contains the Nyquist plot of the transfer function associated with $G$. Based on a similar idea, it is proposed to minimize $r_{d}^{2}$ such that $G$ is $\left\{q_{G} I, s_{G} I, r_{G} I\right\}$-dissipative.

The next lemma shows that verifying that $G$ is $\left\{q_{G} I, s_{G} I, r_{G} I\right\}$-dissipative may be achieved based on an LMI feasibility problem.

Lemma 2:

Let $G$ be described by (8). Let $q_{G}, s_{G}, r_{G} \in \mathbb{R}$ and $q_{G}<0$ be given. Then $G$ is $\left\{q_{G} I, s_{G} I, r_{G} I\right\}$-dissipative if there exists $\boldsymbol{P} \in \mathbb{S}_{4}, \boldsymbol{\Pi} \in \mathbb{D}_{4}$ such that $\boldsymbol{P}>0, \boldsymbol{\Pi}>0$ and

$$
E^{T}\left[\begin{array}{cccccc}
0 & 0 & -\boldsymbol{P} & 0 & 0 & 0 \\
0 & D_{\Delta} \boldsymbol{\Pi} & 0 & -\boldsymbol{\Pi} C_{\Delta} & 0 & 0 \\
-\boldsymbol{P} & 0 & 0 & 0 & 0 & 0 \\
0 & -C_{\Delta} \boldsymbol{\Pi} & 0 & \boldsymbol{\Pi} & 0 & 0 \\
0 & 0 & 0 & 0 & q_{G} I & s_{G} I \\
0 & 0 & 0 & 0 & s_{G} I & r_{G} I
\end{array}\right] E \geq 0
$$

hold where $D_{\Delta}:=C_{\Delta}^{2}-R_{\Delta}^{2}, E:=\left[\begin{array}{ll}E_{1}^{T} & E_{2}^{T}\end{array}\right]^{T}$ and

$$
E_{1}:=\left[\begin{array}{ccc}
A_{G} & A_{\Delta} & B_{p} \\
I & 0 & 0 \\
I & 0 & 0 \\
0 & I & 0
\end{array}\right] \quad E_{2}:=\left[\begin{array}{ccc}
C_{p} & 0 & 0 \\
0 & 0 & I
\end{array}\right]
$$

Let $x_{G}, u \in \mathcal{L}_{2 e}$ be two signals. Pre- and post-muliplying (9) by $\left[\begin{array}{lll}x_{G}^{T} & \left(\Delta x_{G}\right)^{T} & u^{T}\end{array}\right]^{T}$ and its transpose conjugate gives

$$
\begin{array}{r}
{\left[\begin{array}{c}
\dot{x}_{G} \\
x_{G} \\
x_{G} \\
\Delta x_{G}
\end{array}\right]^{T}\left[\begin{array}{cccc}
0 & 0 & -\boldsymbol{P} & 0 \\
0 & D_{\Delta} \boldsymbol{\Pi} & 0 & -\boldsymbol{\Pi} C_{\Delta} \\
-\boldsymbol{P} & 0 & 0 & 0 \\
0 & -C_{\Delta} \boldsymbol{\Pi} & 0 & \boldsymbol{\Pi}
\end{array}\right]\left[\begin{array}{c}
\dot{x}_{G} \\
x_{G} \\
x_{G} \\
\Delta x_{G}
\end{array}\right]} \\
+\left[\begin{array}{l}
y \\
u
\end{array}\right]^{T}\left[\begin{array}{cc}
q_{G} I & s_{G} I \\
s_{G} I & r_{G} I
\end{array}\right]\left[\begin{array}{l}
y \\
u
\end{array}\right] \geq 0
\end{array}
$$

Using similar ideas that for the proof of Lemma 1, the results is obtained by noticing that $\forall \delta \in\left[c_{\delta}-r_{\delta}, c_{\delta}+r_{\delta}\right]$,

$$
\left[\begin{array}{l}
I \\
\Delta
\end{array}\right]^{T}\left[\begin{array}{cc}
D_{\Delta} \Pi & -\Pi C_{\Delta} \\
-C_{\Delta} \Pi & \Pi
\end{array}\right]\left[\begin{array}{l}
I \\
\Delta
\end{array}\right] \leq 0
$$

In order to minimize $r_{d}^{2}$, introduce the slack variable $\lambda$ such that $\lambda \geq r_{d}^{2}$. By the Schur lemma, this is equivalent to

$$
\left[\begin{array}{cc}
\lambda+\frac{r_{G}}{q_{G}} & \frac{s_{G}}{q_{G}} \\
\frac{s_{G}}{q_{G}} & 1
\end{array}\right] \geq 0
$$

Notice that, as there is an infinite number of equivalent $\left\{q_{G}, s_{G}, r_{G}\right\}$ associated with a disk $\left(c_{d}, r_{d}\right)$, one can fix the value of $q_{G}$ to any negative number, e.g. $q_{G}=-1$, without loss of generality. Thus, minimizing $r_{d}^{2}$ such that $G$ is $\left\{q_{G} I, s_{G} I, r_{G} I\right\}$-dissipative, when $q_{G}$ is arbitrarily fixed, may be achieved by solving the following optimization problem with linear objective and LMI constraints:

$$
\underset{\substack{\boldsymbol{\lambda}, \boldsymbol{s}_{\boldsymbol{G}}, \boldsymbol{r}_{G} \in \mathbb{R}, \boldsymbol{P}>0 \in \mathbb{S}_{4}, \boldsymbol{\Pi}>0 \in \mathbb{D}_{4}}}{ } \boldsymbol{\lambda} \text { subject to }(9),(10)
$$

\section{Illustration on the mitigation of a cyber-attack}

Two scenarios are considered. First, an attack by data injection with saturation objective, i.e. $v_{i n}=v_{c}+v_{\max }$, is considered from $t=100 \mathrm{~s}$, where the process is operating at the stationary point $\left(h^{0}, v_{i n}^{0}\right)$ where $h^{0}=$ $\left[\begin{array}{llll}12.40 & 12.70 & 1.592 & 1.455\end{array}\right]^{T}, v_{i n}^{0}=\left[\begin{array}{ll}3.049 & 2.979\end{array}\right]^{T}$. In the second scenario, a dissipation block is interconnected between the process and the network once the attack is detected. It is assumed that the defense mechanisms require $3 \mathrm{~s}$ to detect the attack and trigger the dissipation block interconnection.

The first scenario is illustrated in Fig. 3. It can be observed that, without the defense mechanism, the tanks 1 and 2 overflow. In addition, while the controller adapts its control input to this bias injection to finally come back to the original operating point, this leads to empty the tanks 3 and 4 .

For the second scenario, based on Section V-B with $c_{\delta}=\left[\begin{array}{llll}0.0192 & 0.0189 & 0.0772 & 0.0816\end{array}\right], r_{\delta}=$

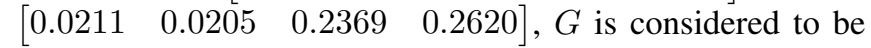




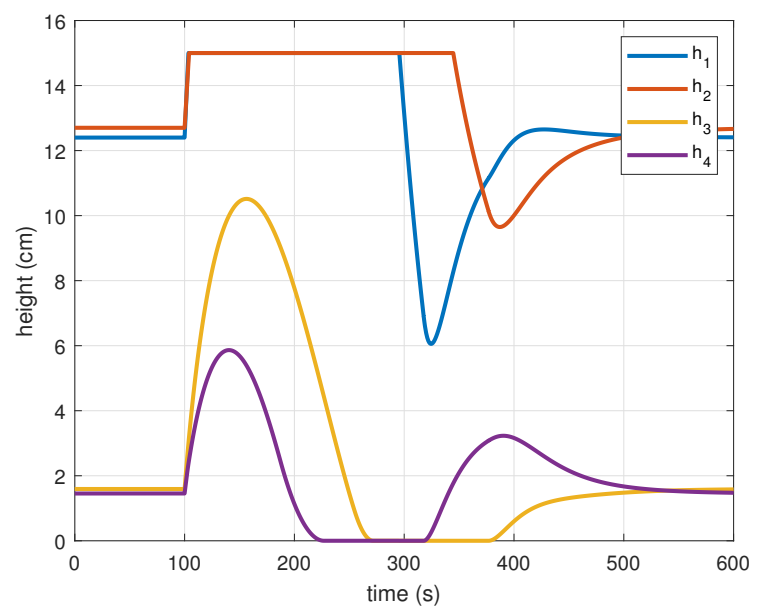

Fig. 3. Upper saturation-injection without defense mechanisms

$\{-I, 1.91 I, 3.46 I\}$-dissipative. Applying Theorem 1 with $Q_{p}=-I, S_{p}=0.5 I, R_{p}=0.25 I$, a dissipation block $M$ is obtained where

$$
\left[\begin{array}{ccc}
A & B_{y} & B_{w} \\
C_{u} & D_{u y} & D_{u w} \\
C_{z} & D_{z y} & D_{z w}
\end{array}\right]=\left[\begin{array}{ccc}
0 & 0 & 0 \\
0 & 0.0520 I & 0.0746 I \\
0 & I & 0
\end{array}\right]
$$

The second scenario is illustrated in Fig. 4. One may observe that the trigger of the dissipation block mechanism mitigates early the anomaly such that the process remains safe.

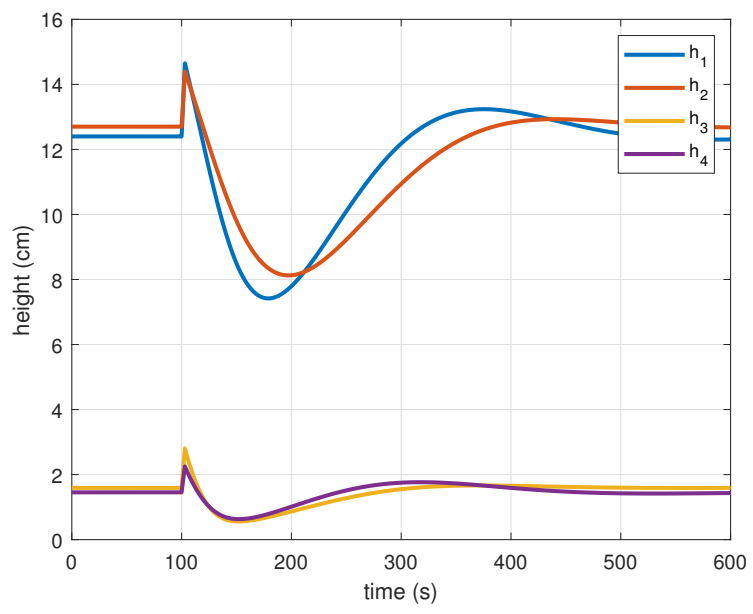

Fig. 4. Upper saturation-injection with dissipation block reconfiguration

\section{CONCLUSION}

In this paper, the synthesis problem of dissipation block for anomaly-tolerant control was addressed. Anomaly is understood here as any kind of attacks, faults or networkinduced disturbances affecting the normal system behavior. It was shown that this problem may be formulated as an LMI feasibility problem. Finally, a numerical example provided promising results for the design of a defense mechanism that ensures resilience with respect to cyber-attacks.
This work paves the way for further investigations on the safety and security of cyber-physical systems based on dissipation block synthesis. In particular, future research directions include practical implementation of such blocks, to explicitly link the global $\{Q, S, R\}$-dissipative characterization with desired resilience properties, or to make an appropriate use of dynamical blocks over static blocks.

\section{REFERENCES}

[1] S. M. Dibaji, M. Pirani, D. Flamholz, A. M. Annaswamy, K. H. Johansson, and A. Chakrabortty, "A Systems and Control Perspective of CPS Security," Annual Reviews in Control, vol. 47, pp. 394-411, 2019.

[2] I. Hwang, S. Kim, Y. Kim, and C. E. Seah, "A Survey of Fault Detection, Isolation, and Reconfiguration Methods," IEEE Transactions on Control Systems Technology, vol. 18, no. 3, pp. 636-653, 2010.

[3] J. Lunze, Control Theory of Digitally Networked Dynamic Systems. Springer, 2014, vol. 1.

[4] Y. Zhang and J. Jiang, "Bibliographical Review on Reconfigurable Fault-Tolerant Control Systems," Annual Reviews in Control, vol. 32, no. 2, pp. 229-252, 2008.

[5] K. Zhou, J. Doyle, and K. Glover, Robust and Optimal Control. New Jersey: Prentice Hall, 1996.

[6] A. J. Van der Schaft, L2-gain and Passivity Techniques in Nonlinear Control. Springer, 2016, vol. Third edition.

[7] A. Perodou, A. Korniienko, G. Scorletti, M. Zarudniev, J. B. David, and I. O'Connor, "Frequency Design of Lossless Passive Electronic Filters: A State-Space Formulation of the Direct Synthesis Approach," IEEE Transactions on Circuits and Systems I: Regular Papers, vol. 68, no. 1, pp. 161-174, 2021.

[8] P. J. Antsaklis, B. Goodwine, V. Gupta, M. J. McCourt, Y. Wang, P. Wu, M. Xia, H. Yu, and F. Zhu, "Control of Cyberphysical Systems using Passivity and Dissipativity based Methods," European Journal of Control, vol. 19, no. 5, pp. 379-388, 2013.

[9] P. Moylan, "Dissipative Systems and Stability," Lecture Notes in collaboration with D. Hill, University of Newcastle, 2014.

[10] A. Perodou, A. Korniienko, M. Zarudniev, and G. Scorletti, "Frequency Design of Interconnected Dissipative Systems: a Unified LMI Approach," in 2018 IEEE Conference on Decision and Control (CDC), 2018, pp. 6250-6255.

[11] N. Kottenstette and P. J. Antsaklis, "Stable Digital Control Networks for Continuous Passive Plants subject to Delays and Data Dropouts," in 2007 46th IEEE Conference on Decision and Control, 2007, pp. 4433-4440.

[12] N. Kottenstette, G. Karsai, and J. Sztipanovits, "A Passivity-based Framework for Resilient Cyber Physical Systems," in 2009 2nd International Symposium on Resilient Control Systems. IEEE, 2009, pp. 43-50.

[13] M. Xia, A. Rahnama, S. Wang, and P. J. Antsaklis, "Control Design Using Passivation for Stability and Performance," IEEE Transactions on Automatic Control, vol. 63, no. 9, pp. 2987-2993, 2018.

[14] M. Xia, P. J. Antsaklis, and V. Gupta, "Passivity Indices and Passivation of Systems with Application to Systems with Input/Output Delay," in 53rd IEEE Conference on Decision and Control, 2014, pp. 783-788.

[15] Y. Yan, M. Xia, A. Rahnama, and P. Antsaklis, "A Passivity-Based Self-Triggered Strategy for Cyber Physical Systems under Denial-ofService Attack," in 2017 IEEE 56th Annual Conference on Decision and Control (CDC), 2017, pp. 6082-6087.

[16] Y. Yan, P. Antsaklis, and V. Gupta, "A Resilient Design for Cyber Physical Systems under Attack," in 2017 American Control Conference (ACC), 2017, pp. 4418-4423.

[17] I. Bessa, V. Puig, and R. M. Palhares, "Passivation Blocks for Fault Tolerant Control of Nonlinear Systems," Automatica, vol. 125, p. 109450, 2021.

[18] S. P. Boyd, L. El Ghaoui, E. Feron, and V. Balakrishnan, Linear Matrix Inequalities in System and Control Theory. SIAM, 1994, vol. 15.

[19] R. A. Horn and C. R. Johnson, Matrix Analysis, 2nd ed. Cambridge University Press, 2012.

[20] K. H. Johansson, "The quadruple-tank process: a multivariable laboratory process with an adjustable zero," IEEE Transactions on Control Systems Technology, vol. 8, no. 3, pp. 456-465, 2000. 